\title{
A method to assess the accuracy of Pseudo-random Number Sampling Methods from evacuation datasets
}

\author{
Cuesta, A. (cuestaar@unican.es) ${ }^{1 *}$,Alvear, D. (alveard@unican.es) $)^{1}$,_Abreu, O. \\ (abreuo@unican.es) $)^{1}$ and Alonso, V. (v.alonso-gutierrez@imperial.ac.uk) ${ }^{2}$ \\ ${ }^{1}$ Departamento de Transportes y Tecnología de Proyectos y Procesos, Universidad de Cantabria \\ Fax: +34942 202276 \\ Tel: +34942201826 \\ ${ }^{2}$ Department of Mechanical Engineering, Imperial College London
}

\begin{abstract}
We propose a method for assessing the accuracy of Pseudo-random Number Sampling Methods (PNSMs) for evacuation modelling purposes. It consists of a systematic comparison between experimental and generated distributions. The calculated weighted relative error $\left(E_{w_{-} r e l}\right)$ is based on the statistical parameters as central moments (mean, standard deviation, skewness and kurtosis) to shape the distribution. The case study involves the Box Muller transform, the KernelEpanechnikov, the Kernel-Gaussian and the Picewise linear generating samples from eight evacuation datasets fitted against normal, lognormal and uniform distributions. Keeping in mind that the Bos Muller method has two potential sources of error (i.e. distribution fitting and sampling), this method produces plausible results when generating samples from the three types of distributions ( $E_{w_{-} r e l}<0.30$ for normal, lognormal and uniform distributions). We also fund that the Kernel Gaussian and the Kernel Epanechnikov methods are well accurate in generating samples from normal distributions $\left(E_{w_{-} r e l}<0.1\right)$ but potentially inaccurate when generating samples from uniform and lognormal distributions $\left(E_{w_{-} r e l}>0.80\right)$. Results suggest that the Picewise linear is the most accurate method $\left(E_{w_{-} r e l}=0.01\right.$ normal; $E_{w_{-} r e l}=0.04$ lognormal; $E_{w_{-} r e l}=0.009$ uniform). This method has the advantage of sampling directly from empirical datasets i.e. no previous distribution fitting is needed. While the proposed method is used here for evacuation modelling, it can be extended to other fire safety engineering applications.
\end{abstract}

Keywords: Evacuation modelling, Pseudo-random Number Sampling Methods, Empirical evacuation data

\section{Introduction}

Computer models are being used to simulate, visualize, manipulate and gain information of evacuation process. It is greatly accepted that evacuation is a random process due to the variability in occupant characteristics, reactions and behaviours [1-4]. A key point is the way current and future computer evacuation models address this randomness. Evacuation models may be based upon "best fit curves" to represent a set of data. In other words, a distribution giving a close fit is supposed to lead to more accurate predictions. This involves two main issues that can increase or decrease the deviation of the behaviour of the potential-actual evacuation from the desired behaviour of the simulated evacuation.

The first issue is the selection of the appropriate Probability Density Function (PDF) that suits the data well. Most computer evacuation models allow the user to introduce random inputs by a 
defined set of PDFs. Normal, lognormal and uniform are the most commonly used distributions [5-13]. Also, other distributions (exponential, polynomial, gamma, beta, triangular, Weibull and Gumbel) have been observed in models [5, 8-13]. Some models allow the users to build a PDF based on their own sampling [6, 9, 11-14]. In addition, data to shape the random variables in evacuation models is scarce and limited. We can find a number of works in the literature collecting data [15]. Datasets are usually presented in the following format: average, standard deviation, maximum and minimum values. Information such as sample sizes, data collection techniques and the background conditions are occasionally provided $[16,17]$. Nevertheless, in most cases, neither the raw data is available nor the type of PDF is defined. Within this context, the user has two options to configure the model for evacuation analysis: 1) choose a PDF (from a library of standard distributions available in the model) and use the parameters from the literature (mean, standard deviation, max. and min. values) or 2) process and use empirical data. The first choice is commonly applied. For instance, the selection of positively skewed distributions for pre-evacuation times and normal distributions for walking speeds is suggested in [18-20]. Another approach suggests also the use of uniform distributions [21]. The second one is the most plausible choice (process and use empirical data) as it uses distributions derived from the scenario of analysis.

The second issue is related with the degree to which a random variable (or a set of random variables) within a computer evacuation model conforms to a true value or referent. In other words, the way computer evacuation models generate pseudo-random numbers that are distributed according to a given probability distribution. Although some works have attempted to address uncertainty in evacuation modelling [2, 22, 23], the analysis of Pseudo-random Number Sampling Methods (PNSMs) potentially applied to evacuation models has been ignored. Furthermore, to the author's knowledge, current evacuation models do not specify the mathematical and computational basis for generating stochastic inputs of the variables. It is well known that the applications for random numbers cover several fields such as simulation [24], chemistry [25] or cryptography [26]. There are several methods for the assessment of the accuracy and robustness of pseudo-random sampling in the literature [26,27]. Studies have focused on randomness testing to analyse whether or not the generated data by random number generator (RNG) is "patternless" and therefore the RNG used is suitable for a particular application. Evacuation modellers can choose validated RNGs from reputable sources. The PNSMs are typically based on the availability of a RNG producing numbers $X$ that are uniformly distributed. Computational algorithms are then used to manipulate a single random variate, $X$, or often several such variates, into a new random variate $Y$ such that these values have the required distribution. This paper focuses on PNSMs defined here as a transformation from an independent uniform sample or samples $[0,1]$ to a sample of another given distribution.

Given the importance of using random variables and understanding the issues associated with the inputs and the intrinsic algorithms of models, our primary objective in this paper is to provide a comprehensive method for assessing the accuracy of different PNSMs when sampling from evacuation datasets. The paper is intended for those involved in evacuation modelling - from model users to model developers - to better understand and improve current and future developments.

\section{Method}

This section presents the analysis of different PNSMs when sampling from evacuation datasets. It includes an overview of the proposed method, a description of the evacuation datasets, the PNSMs selected and the description of the software developed for the application case. 


\subsection{Overview of the proposed method}

Sampling in the statistical sense means to retrieve a value. The value we get is decided by the probability distribution we are trying to sample from. Accuracy is defined here as the closeness of a modelled/computed value to its "true" value. The "true" value is the value it would have if we had perfect information. Hence, we establish the following postulate: the smaller the relative error between the shape of the observed distribution and the shape of the generated distribution, the more accurate the PNSM used.

Figure 1 shows an overview of the proposed method to assess the accuracy of PNSMs for evacuation modelling purposes. It mainly consists of a systematic comparison between experimental and generated distributions including both Analytical and Empirical PNSMs. Analytical PNSMs are based on algebraic equations using a RNG (Random Number Generator). Empirical PNSMs are other non-parametric alternatives for sampling of a random variable based on observed data. The accuracy of PNSMs is given by the following two steps. The first step is the calculation of the relative error of each statistical parameter (mean, standard deviation, skewness and kurtosis):

$$
\left.E_{\text {rel }}\right|_{m}=\left|\frac{x_{r}-x_{g}}{x_{r}}\right|
$$

Where:

$E_{\text {rel }}$ - relative error of the parameter x;

$x_{r}$ - statistical parameter from the observed variable;

$x_{g}$ - statistical parameter from the generated variable;

$m$ - the PNSM used.

The second step is the calculation of the weighted relative error of the statistical parameters:

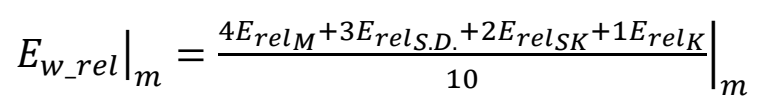

Where:

$E_{w_{-} r e l}$ - weighted relative error;

$E_{\mathrm{rel}_{M}}$ - relative error of the mean value;

$E_{r e l_{S . D .}}$ - relative error of the standard deviation value;

$E_{\text {rel }}$ - relative error of the skewness value;

$E_{r e l_{K}}$ - relative error of the kurtosis value.

The coefficients in equation (2) are based on the order of statistical parameters as central moments and their importance as estimators of a given distribution (4 mean, 3 standard deviation, 2 skewness and 1 kurtosis). 


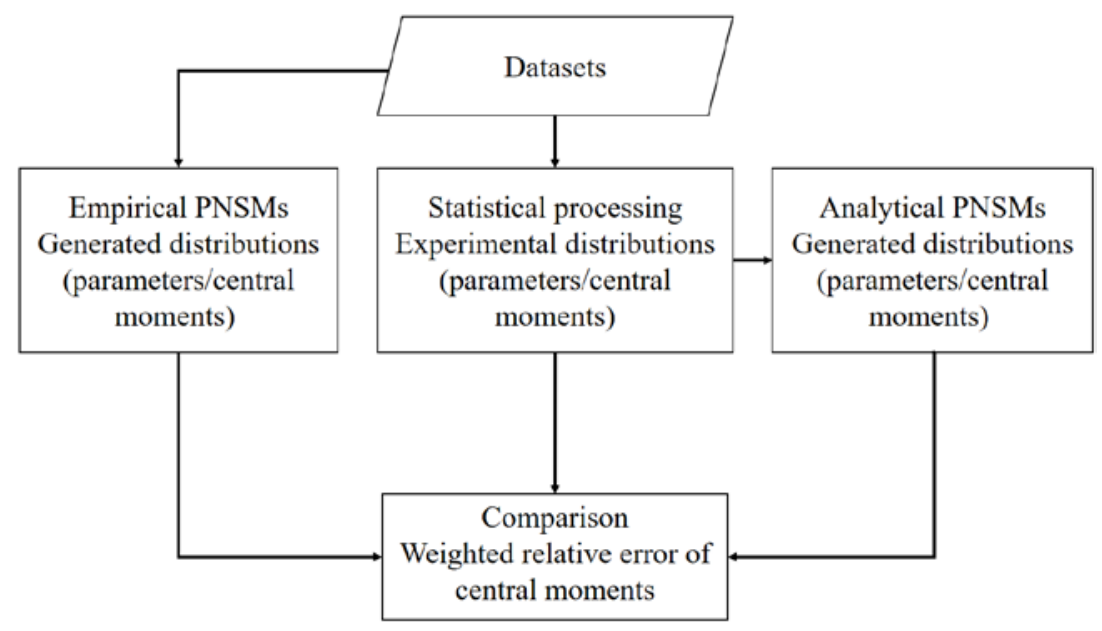

Fig. 1. Overview of the proposed method for assessing the accuracy of different PNSMs.

\subsection{Datasets}

As mentioned, data to shape the random variables in evacuation models is scarce and limited. A deliberate effort was made to use representative datasets derived from evacuation experiments and fire drills conducted in different evacuation scenarios: passenger train, school building and transport station. Given the practical scope of this paper, datasets corresponded to several variables, with different sample sizes and fitted against different distributions. The datasets used are already published and further information can be found in the references of each experiment. However, the data are presented in this section independently, given the different possible uses of this data. We also describe the collection techniques and the background conditions to better understand how the data was obtained.

The fire drills in passenger trains consisted of a relocation procedure inside the train (coach by coach) and the evacuation by a single exit once the train has stopped (see Figures 2 and 3). The Drill 1 and the Drill 2 involved 83 and 218 participants respectively. Participants were railroad employees. They had no luggage, but could gather personal belongings and carry them out during the drills [28, 29]. Video-cameras were used to capture passengers' behaviours. Video images were collected at a frequency of 30 frames/sec and were analysed using the Avidemux 2.5.2 software that allows the footage to be manually advanced frame by frame. The data collected consisted of the response time ( $\left.t_{\text {res } 1}\right)$, the stop time ( $\left.t_{\text {stop }}\right)$, the exit time ( texit $)$ and the walking speed within the aisle ( $S_{a i s l e}$ ). The response time (collected from Drill 1 ) is defined as the time from the moment at which the evacuation is announced (alarm) through public address system until the passenger is stood up and starts the preparation activities. The stop time (collected from Drill 1) is the time spent by some passengers blocking the aisle to collect belongings, wait others, etc. The exit time (collected from Drill 2) is the time spent by each passenger to negotiate the train steps. Finally, the walking speed within the aisle (collected from Drill 2) is defined as the unimpeded walking speed of each passenger through the aisle inside the train. Collected from Drill 2. Figure 4 shows the histograms of the observed data. 


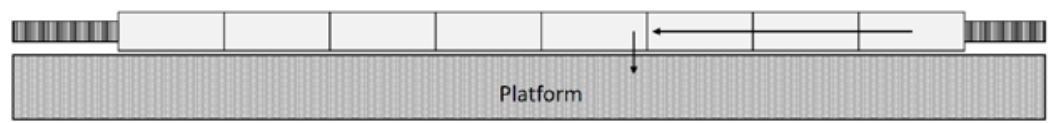

Evacuation procedure of Drill 2

Tunel

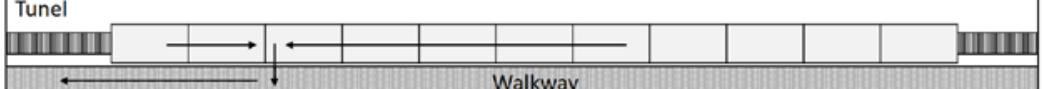

Fig. 2. Evacuation procedures applied to Drill 1 and Drill 2. Only one exit was used in both evacuations.
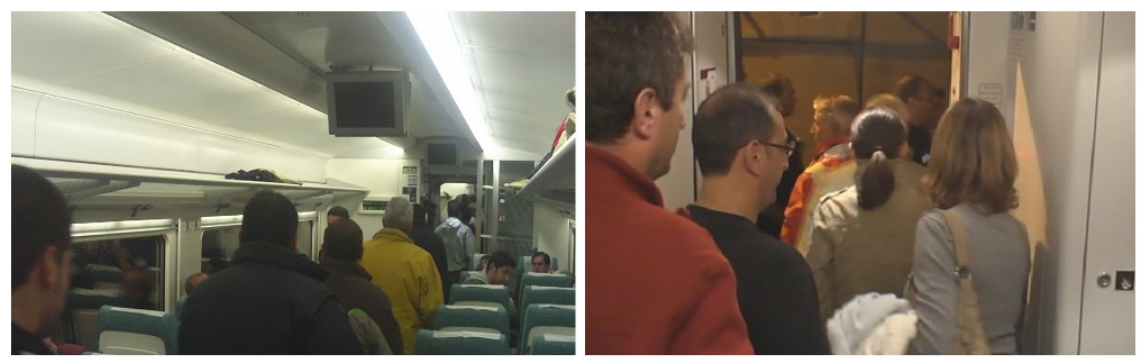

Fig. 3. Relocation procedure onboard the train and evacuation of passengers to the walkway inside the tunnel during the evacuation Drill 2.

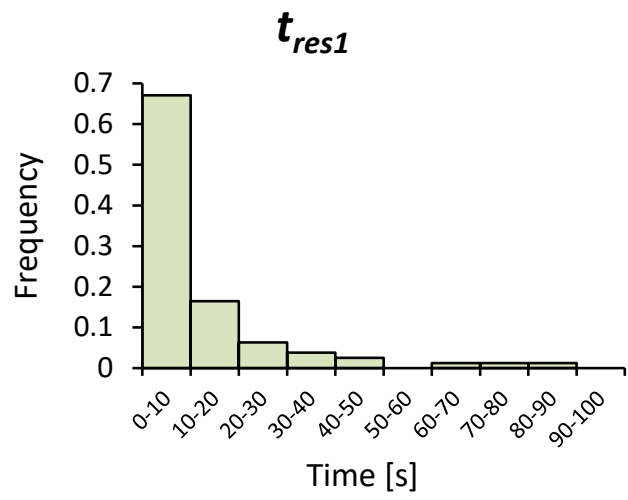

a) Response time

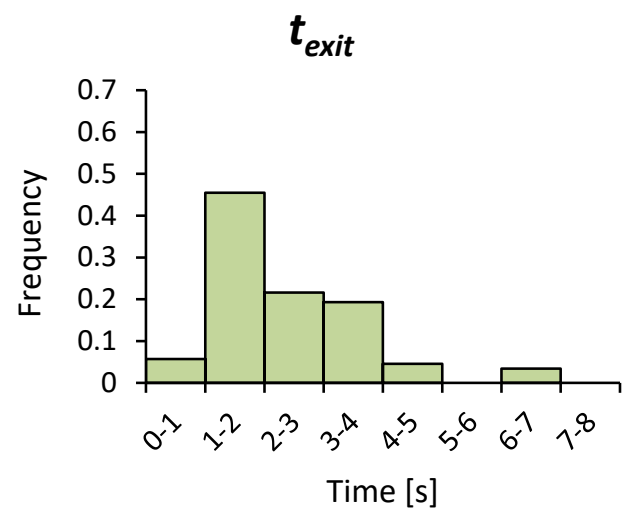

c) Exit time

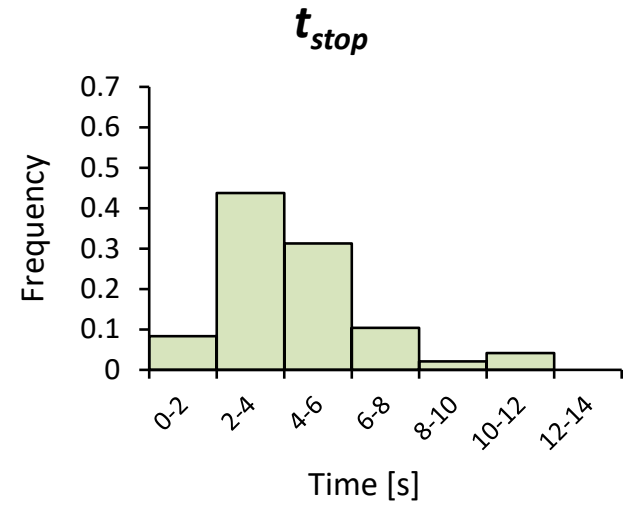

b) Stop time

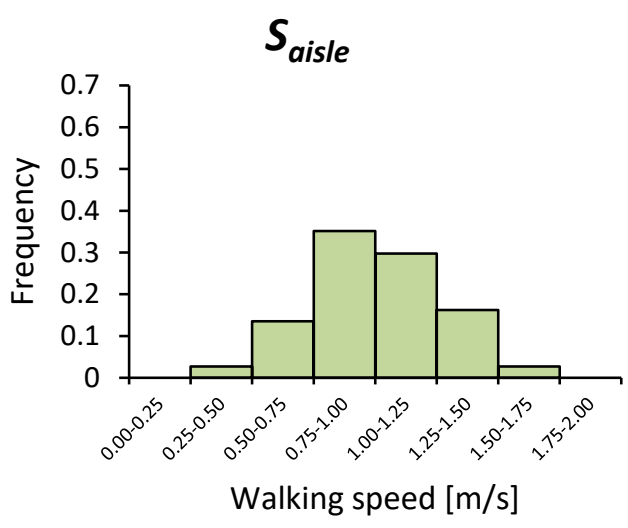

d) Walking speed within the aisle

Fig. 4. Histograms of observed variables during evacuation from passenger trains.

The analysis also included datasets collected during a semi-announced evacuation trial in a school building [16, 17] (i.e. students had no warning, while the teaching staff had some prior warning in the trial). The video-cameras were positioned at ceiling height to improve the vantage point and 
reduce the impact of the cameras on the evacuation process (see Figure 5). Video images were analysed frame by frame using the Avidemux 2.5.2 software as well. Figure 6 shows the histograms of the observed response times and walking speeds downstairs. Datasets consist of the response time ( $\left.t_{r e s 2}\right)$ defined as the time from the alarm to the child is stood up and starts evacuation movement (students from 6 to 7 years old) and the walking speed downstairs ( $\left.S_{\text {stair }}\right)$ defined as walking speed of each child on a stair (students from 12 to 16 years old).
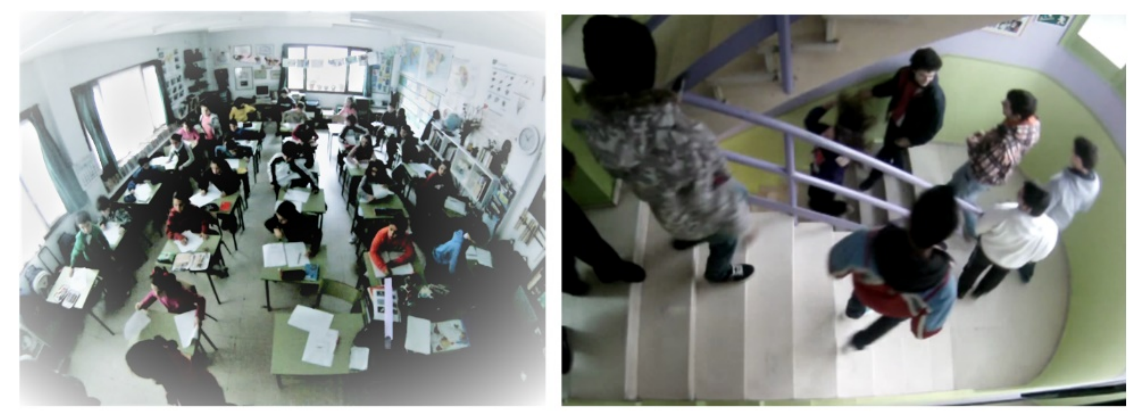

Fig. 5. Snapshots of students (6-7 years old) inside a classroom when the alarm went off and students (12-16 years old) walking downstairs during the evacuation trial in the school building.

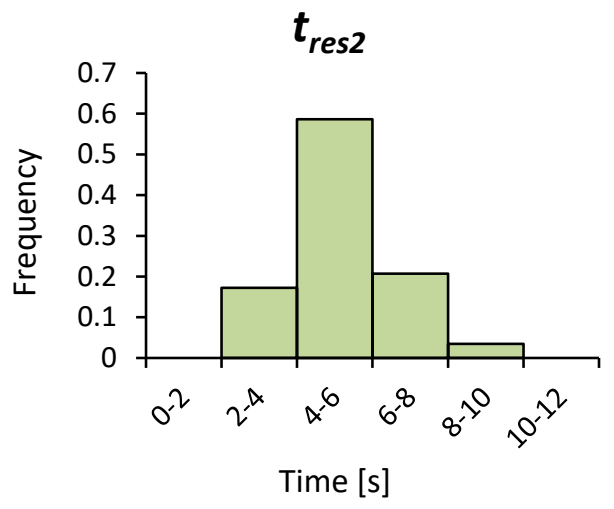

a) Response time

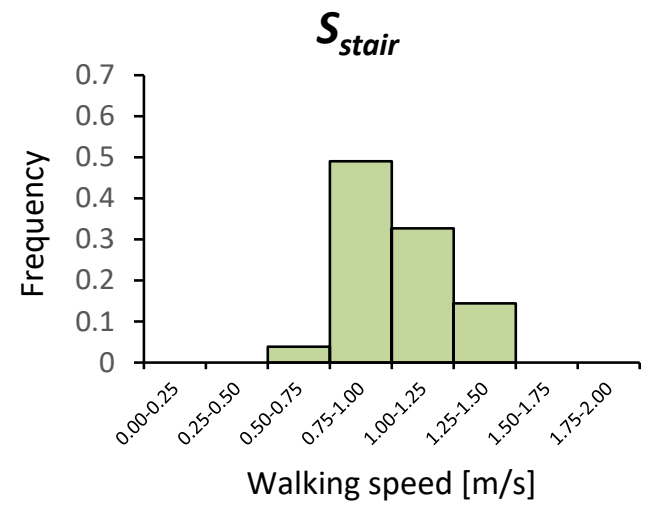

b) Walking speed downstairs

Fig. 6. Histograms of observed response time in a classroom (students 6-7 years old) and walking speed downstairs (students 12-13 year sold) during the evacuation trial in the school building.

We also use datasets from evacuation trials in an underground bus concourse [30]. Participants were recruited by a casting agency. They were told that they were going to take part in transport comfort and mobility tests. Each participant was given a slip of paper with the number of a boarding gate to go and was instructed to remain there. When all participants were at their respective location, the alarm went off. The trials were finished when all participants had evacuated the bus concourse trough the available emergency exits (see Figure 7).

Two response time data samples were selected from the 15 trials conducted, namely tres3 and tres4. Here, the response is also defined as the time from the sounding of the alarm (voice message) to deliberate evacuation movement(s). Figure 8 shows the histograms of the response time variables (tres3 and $t_{\text {res } 4}$ ).

Table 1 summarizes the statistical characteristics of the data samples used (mean, standard deviation, kurtosis and skewness). 

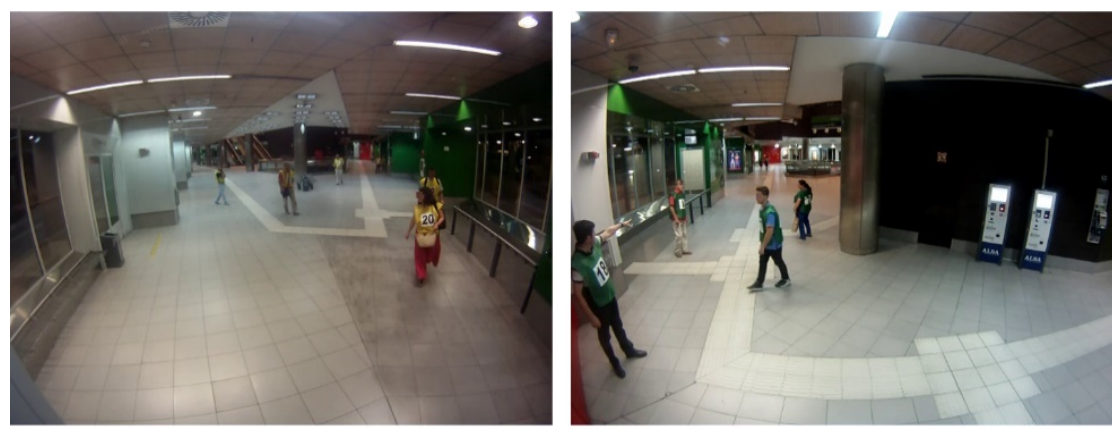

Figure 7. Participants evacuating the bus concourse during the evacuation experiments in a multimodal station.
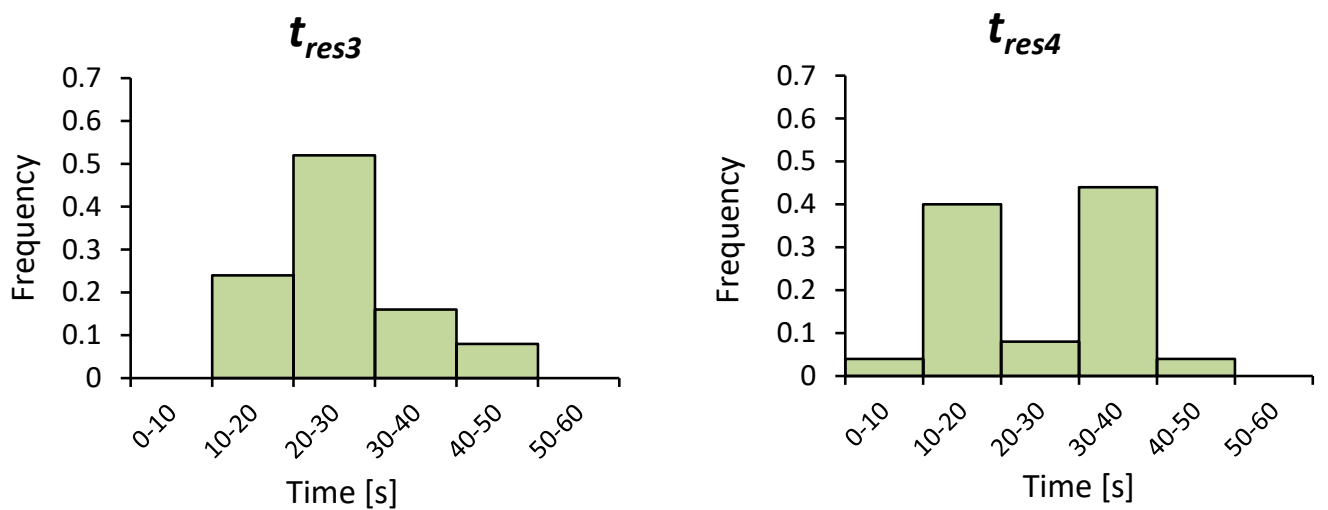

Fig.8. Histograms of observed response times in the station.

Table 1. Statistical characteristics of the observed evacuation datasets used for the analysis.

\begin{tabular}{lcccccccc}
\hline Scenario & \multicolumn{3}{c}{ Passenger trains } & \multicolumn{2}{c}{ School } & \multicolumn{2}{c}{ Station } \\
\hline Variable & $\boldsymbol{t}_{\text {res1 }}$ & $\boldsymbol{t}_{\text {stop }}$ & $\boldsymbol{S}_{\text {aisle }}$ & $\boldsymbol{t}_{\text {exit }}$ & $\boldsymbol{t}_{\text {res2 }}$ & $\boldsymbol{S}_{\text {stair }}$ & $\boldsymbol{t}_{\text {res3 }}$ & $\boldsymbol{t}_{\text {res4 }}$ \\
\hline \# data-points & 79 & 48 & 88 & 74 & 29 & 104 & 25 & 25 \\
\hline Mean & 11.91 & 4.37 & 0.99 & 2.27 & 5.27 & 1.00 & 26.44 & 26.51 \\
\hline Standard deviation & 16.25 & 2.14 & 0.27 & 1.27 & 1.26 & 0.18 & 7.63 & 10.72 \\
\hline Kurtosis & 2.74 & 1.32 & 0.20 & 1.45 & 0.38 & 0.37 & -0.14 & -0.14 \\
\hline Skewness & 8.07 & 1.98 & -0.60 & 2.48 & -0.22 & -0.55 & -0.51 & -1.59 \\
\hline
\end{tabular}

The statistical treatment of the samples described allowed the probability distribution laws to be obtained. For assessing whether a given distribution is suited to dataset, the following Goodnessof-fit tests and their underlying measures were used: the D' Agostino's K-square normality test (Omnibus $\mathrm{K}^{2}$ statistic) [31,32], the Hypothetical log-normal test applying the normality test $\left(y_{i}=\ln \left(x_{i}\right)\right)$ and the Modified Anderson-Darling test for Uniformity (modified normality test) [33]. Specific tests were considered because they have more power.

Table 2 shows the results of the Goodness-of-fit tests conducted. Data from $t_{\text {res } 1, ~} t_{\text {stop }}$ and $t_{\text {exit }}$ variables fitted against lognormal distribution, and data of $S_{a i s l e}, S_{\text {stair }}$ and $t_{\text {res2 } 2 \text { variables fitted }}$ against normal distribution and data of $t_{r e s} 3$ and $t_{r e s} 4$ variables fitted against uniform distribution.

Table 2. Results of the Goodness-of-fit tests for the selected evacuation variables. 


\begin{tabular}{|c|c|c|c|c|c|c|c|c|}
\hline \multirow{2}{*}{$\begin{array}{l}\text { Scenario } \\
\text { Variable }\end{array}$} & \multicolumn{4}{|c|}{ Passenger trains } & \multicolumn{2}{|c|}{ School } & \multicolumn{2}{|c|}{ Station } \\
\hline & $t_{\text {res1 }}$ & $t_{\text {stop }}$ & $t_{\text {exit }}$ & $S_{\text {aisle }}$ & $t_{\text {res2 }}$ & $S_{\text {stair }}$ & $t_{\text {res3 }}$ & $t_{\text {res } 4}$ \\
\hline \# data-points & 79 & 48 & 74 & 88 & 29 & 104 & 25 & 25 \\
\hline Test & \multicolumn{3}{|c|}{$\begin{array}{l}\text { D’ Agostino’s K }{ }^{2} \\
\text { normality test }\end{array}$} & \multicolumn{3}{|c|}{$\begin{array}{c}\text { Hypothetical log-normal test } \\
\text { applying D’ Agostino's K }{ }^{2} \\
\text { normality test }\end{array}$} & \multicolumn{2}{|c|}{ Anderson-Darling } \\
\hline Critical value $(\alpha=0.05)$ & \multicolumn{6}{|c|}{$\mathrm{K}_{\text {(crit) }}^{2}=5.991$} & \multicolumn{2}{|c|}{$\mathrm{A}_{\text {(crit) }}^{2}=2.492$} \\
\hline Statistic & 0.816 & 0.088 & 4.415 & 2.031 & 1.012 & 4.434 & 1.246 & 1.632 \\
\hline Distribution Law^$\wedge$ & $\mathrm{LN}$ & $\mathrm{LN}$ & $\mathrm{LN}$ & $\mathrm{N}$ & $\mathrm{N}$ & $\mathrm{N}$ & $\mathrm{U}$ & $\mathrm{U}$ \\
\hline
\end{tabular}

^ Distribution Law: fitted against Normal (N), Lognormal (LN) or Uniform (U) distribution.

\subsection{Selected PNSMs}

As mentioned, PNSMs are defined as a transformation given a source of uniformly distributed random numbers $[0,1]$ generated by RNGs to a sample of another given distribution. There are several PNSMs in the literature. Note that the aim of this study was to test the validity of the proposed method. The criteria for selecting the PNSMs for this analysis was based on authors judgment but considering representative methods of both analytical and empirical approaches for generating samples from normal, lognormal and uniform distributions. Note that the proposed method can be applied to other PNSMs.

\subsubsection{Analytical methods}

\section{Generation of normally distributed numbers}

Analytical methods are based on algebraic equations to generate normal random variables involving the use of a RNG (Random Number Generator) that provides a sequence of uniform random variables on $[0,1]$. There are 4 main ways of converting them into $N(0,1)$ : the Box-Muller method [34], the Inverse CDF transformation [34], the Marsaglia's polar method [36] and the Marsaglia's ziggurat method [37]. Although all available PNSMs are suitable for the proposed method, we selected the Box Muller transform. This method was developed as more computationally efficient alternative to the inverse transform sampling method and it is commonly used for generating normally and log normally distributed numbers and very similar to Marsaglia's polar method.

The Box Muller takes $R_{1}$ and $R_{2}$, two independent uniformly distributed random variables on $[0,1]$ and defines:

$$
z=\sqrt{-2 \ln \left(R_{1}\right)} \cdot \cos \left(2 \pi R_{2}\right)
$$

Where:

$Z$ - random number normally distributed (mean 0 and standard deviation 1 );

$R_{1}, R_{2}$ - two different random numbers uniformly distributed between 0 and 1 (generated by the RNG). 
Then, to generate a random input from a normal distribution with specific statistical parameters:

$$
y=s \cdot z+m
$$

Where:

$y$ - random variable from a specific normal distribution (mean $m$ and standard deviation $s$ );

$m$ - mean value of the normal distribution;

$s$ - standard deviation of the normal distribution;

\section{Generation of log-normally distributed numbers}

It is well known that a lognormal distribution function is a continuous probability distribution of a random variable with a logarithm normally distributed:

$$
x=\ln (y)
$$

Where:

$x$ - random variable from a specific lognormal distribution (mean $\mu$ and standard deviation $\sigma$ );

$y$ - random variable from the specific normal distribution (mean $m$ and standard deviation $s$ ).

Based on this relationship, the method defined in the previous section can be applied for generating Lognormal distributions. However, the following transformations will be employed to get the mean $m$ and standard deviation $s$ values from the normal distribution.

$$
\begin{aligned}
& m=\ln \mu-\frac{1}{2 \ln \left(1+\frac{\sigma^{2}}{\mu^{2}}\right)} \\
& s=\sqrt{\ln \left(1+\frac{\sigma^{2}}{\mu^{2}}\right)}
\end{aligned}
$$

Where:

$\mu$ - mean value from the lognormal distribution;

$\sigma$ - standard deviation value from the lognormal distribution;

$m$ - mean value from the normal distribution (obtained from the Lognormal distribution);

$s$ - standard deviation value from the normal distribution (obtained from the lognormal distribution).

\section{Generation of uniformly distributed numbers}

The selected method is based on the RNG to generate a random number $R$ uniformly distributed $[0,1]$. The following transformation is considered to obtain a random input from a uniform distribution between a minimum value $a$ and a maximum value $b$ :

$$
y=R \cdot(b-a)+a
$$

Where:

$y$ - Number uniformly distributed between $a$ and $b$. 


\subsubsection{Empirical methods}

There are also non-parametric alternatives to generate pseudo-random numbers directly from evacuation datasets, namely Empirical methods. In other words, no previous distribution fitting is needed. Here we describe those methods selected for the case study; the Piecewise linear [38], the Kernel-Gaussian and the Kernel-Epanechnikov [39].

\section{$\underline{\text { Piecewise linear function }}$}

Given a sample data $x$ - from drill, experiment or actual emergency-:

$$
x=\left\{x_{0}, x_{1}, x_{2}, \ldots, x_{n-1}\right\}
$$

The Piecewise linear function $\mathrm{F}(\mathrm{x})$ is composed of straight-line sections (see Figure 9) with CDF values calculated by

$\left.f_{i}=(i+0.5) / n \quad 0 \leq i \leq n-1\right)$

and a random number $R i$ uniformly distributed on interval $[0,1]$ is possible to choose a series $\mathrm{k}$, such that:

$$
f_{k-1} \leq R_{i} \leq f_{k}
$$

the expression for generating a random variable $y$ from the sample data $\mathrm{x}$ is:

$$
y_{i}=x_{k-1}+\left(x_{k}-x_{k-1}\right)\left(n R_{i}+k+0.5\right)
$$

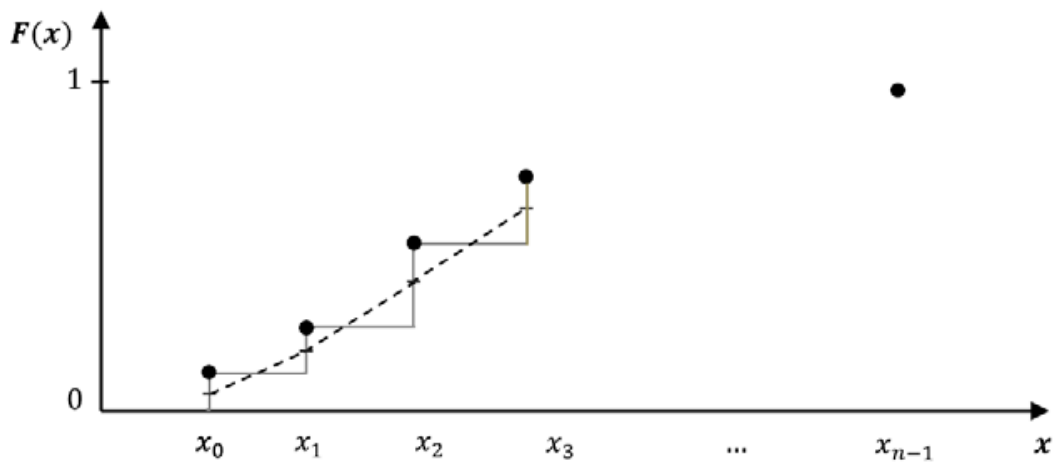

Fig. 9. Piecewise linear estimation for $X$ variable.

\section{$\underline{\text { Kernel estimation }}$}

Equation (10) shows the general expression for the generation of a random input by using a Kernel estimation:

$$
\widehat{f_{n}}(x)=\frac{1}{n} \sum_{i=1}^{n} K_{h}\left(x-x_{i}\right)
$$

Where:

$n$ - the size of the initial sample whose PDF is estimated;

$x=\left\{x_{0}, x_{1}, x_{2}, \ldots, x_{n-1}\right\}-$ the initial sample; 
$K_{h}$ - Kernel function;

$h$ - bandwidth.

and

$$
K_{h}\left(x-x_{i}\right)=\frac{1}{n h} K\left(\frac{x-x_{i}}{h}\right)
$$

We propose the analysis of the following kernel functions:

Gaussian:

$$
K(U)=\frac{1}{\sqrt{2 \pi}} e^{-\frac{1}{2} u^{2}}
$$

Epanechnikov.

$$
K(U)=\frac{3}{4}\left(1-U^{2}\right) \cdot 1_{(|U| \leq 1)}
$$

Based on the previous equation, the generation of stochastic inputs from an estimated PDF through Kernel is implemented to generate a random number $j$ between $[1, \mathrm{n}]$ and to generate a random number fitted to the Kernel function - Gaussian or Epanechnikov - centred in $x_{j}$. Firstly, the bandwidth is obtained according to Silverman's rule of thumb to minimize the AMISE (asymptotic mean integrated squared error) as [40]:

$$
\begin{aligned}
& h=1.06 \frac{s}{n^{0.2}}-\text { Gaussian } \\
& h=2.34 \frac{s}{n^{0.2}}-\text { Epanechnikov }
\end{aligned}
$$

Secondly, the random input $y_{i}$ using the corresponding Kernel is obtained as Gaussian and Epanechnikov. For Gaussian:

$y_{i}=h N_{i}+x_{j}$

Where:

$N_{i}$ - random number normally distributed between 0 and 1 (using Box-Muller transform).

For Epanechnikov, considering:

$K(U)=K\left(\frac{x-x_{i}}{h}\right)=\frac{3}{4 h}\left(1-\frac{\left(x-x_{i}\right)^{2}}{h^{2}}\right)$

Based on equation (18) and solving the corresponding integral, the generation of the random input $y_{i}$ using Kernel -Epanechnikov is completed thusly:

$y_{i}=h E_{i}+x_{j}$

Where:

$E_{i}=2 \sin \left[\frac{1}{3} \arcsin \left(2 R_{1 i}-1\right)\right]$;

$R_{1 i}$ - Random number normally distributed between 0 and 1 (using Box-Muller transform). 


\subsection{Software for PNSMs accuracy}

As mentioned, the smaller the relative error between the shape of the observed distribution and the shape of the generated distribution, the more accurate the PNSM. To test this, we developed a software in C\# Object Oriented Programming language by using Visual Studio. As Figure 10 shows, the software operates following four main steps. In the first step, the software imports and processes statistically the experiment data. The software provides distribution fitting and estimated central moments. It uses the following Goodness-of-fit tests $(\alpha=0.05)$ : D' Agostino's K-square normality test, Hypothetical log-normal test applying the normality test and Anderson-Darling uniformity test (modified normality test). In the second step, the software generates samples of data using each PNSM described in section 2.3 (i.e. based on distribution parameters). The RNG used by the software is based on the modified version of Donald E. Knut's subtractive random generator algorithm [24]. In the third step, the software calculates the relative error for the statistical parameters described in equation (1). Finally, in the fourth steps the software calculates the weighted relative error for each PNSM based on the order of the statistical parameters as central moments (4 mean, 3 standard deviation, 2 skewness and 1 kurtosis) as described in equation (2).

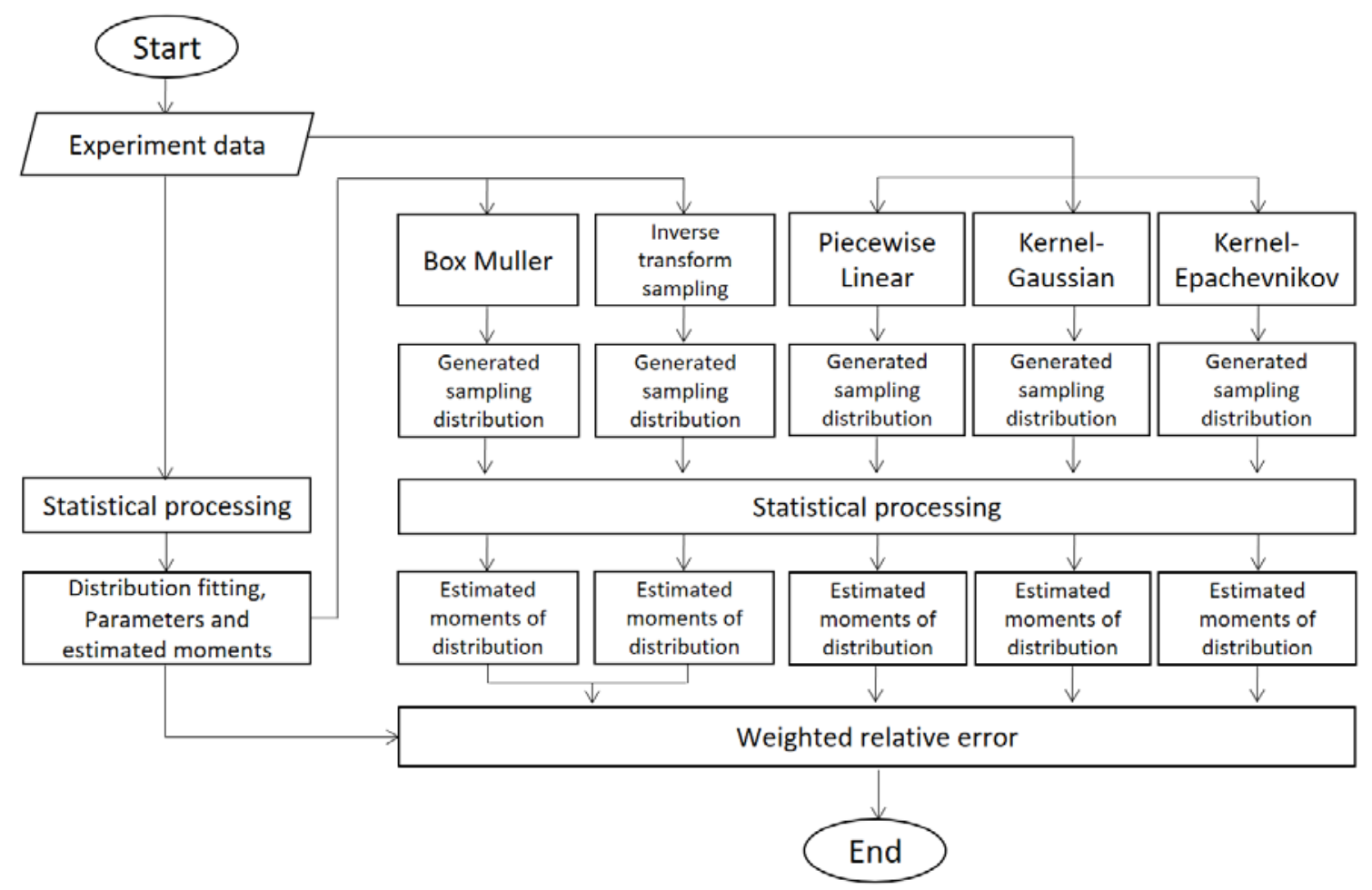

Fig. 10. Model flowchart for assessing the accuracy of different PNSMs.

\section{Results}

The software allows generating preudo-random numbers with different sample sizes. A previous analysis was conducted to check the sensitivity of results to number of data under consideration. Sample sizes of 250, 500, 1.000 and 1.500 were generated to do an explanatory check of if (and to what extend) this affects results. The absolute errors for the weighted relative errors produced when compare 250 and 1.500 are presented in Table 3. It is apparent from Table 3 that very few differences are produced with different sample sizes except for the variable $t_{\text {res } 1}$ by using the Box Muller method. Despite the changes in the generated sample size is out of the purpose of this paper, this comparison helps drawing more solid conclusions in the following results. 
According to the Law of Large Numbers, the larger the sample size the narrower the confidence interval of the central moments (mean, standard deviation, skewness and kurtosis). The dedicated software was used for generating samples using each PNSMs: the Box-Muller, the Piecewise linear, the Kernel-Gaussian and the Kernel-Epanechnikov. The results presented here correspond to a generated sample sizes of 1.500 values. The distributions were statistically analysed and the relative errors for the central moments were calculated to compare the accuracy of the selected PNSMs.

Table 3. Absolute errors of the weighted relative errors comparing generated samples of 250 and 1.500 values $(B M=$ Box Muller transform; $P L=$ Piecewise linear; $K G=$ Kernel-Gaussian; $K E=$ KernelEpanechnikov). Cells in grey show relative errors higher than 1.

\begin{tabular}{ccccccccc}
\hline PNSM & $\boldsymbol{t}_{\text {res1 }}$ & $\boldsymbol{t}_{\text {res2 }}$ & $\boldsymbol{t}_{\text {res3 }}$ & $\boldsymbol{t}_{\text {res4 }}$ & $\boldsymbol{S}_{\text {aisle }}$ & $\boldsymbol{S}_{\text {stair }}$ & $\boldsymbol{t}_{\text {exit }}$ & $\boldsymbol{t}_{\text {stop }}$ \\
\hline $\mathrm{BM}$ & 0.491 & 0.009 & 0.007 & 0.002 & 0.002 & 0.004 & 0.059 & 0.037 \\
\hline $\mathrm{PL}$ & 0.007 & 0.000 & 0.002 & 0.005 & 0.000 & 0.002 & 0.008 & 0.004 \\
\hline $\mathrm{KG}$ & 0.003 & 0.002 & 0.008 & 0.001 & 0.002 & 0.003 & 0.004 & 0.000 \\
\hline $\mathrm{KE}$ & 0.004 & 0.003 & 0.004 & 0.004 & 0.001 & 0.000 & 0.003 & 0.000 \\
\hline
\end{tabular}

Table 4 shows the relative errors of central moments. All PNSMs produce accurate (and precise) values for the means with relative errors between 0 and 0.030 . The higher relative errors are produced by Kernel-Gaussian and the Kernel-Epanechnikov methods for the standard deviations of response time variables $t_{r e s 1}, t_{\text {res3 } 3}$ and $t_{\text {res } 4}$ with values ranged from 3.465 to 6.337. This is possibly due to the use of Silverman's rule of thumb to minimize the AMISE (asymptotic mean integrated squared error). While this rule of thumb is easy to compute, it should be used with caution as it can yield widely inaccurate estimates when the density is not close to being normal.

Table 4. Relative errors of central moments (mean, standard deviation, skewness and kurtosis) for the PNSMs (BM = Box Muller transform; PL = Piecewise linear; $K G=$ Kernel-Gaussian; KE = Kernel-Epanechnikov). Cells in grey show relative errors higher than 1.

\begin{tabular}{ccc|cc}
\hline \multicolumn{5}{c}{ Response time } \\
\hline & $\left.\boldsymbol{t}_{\text {res1 }}\right)$ \\
\hline $\boldsymbol{E}_{\text {relM }}$ & 0.001 & 0.000 & 0.030 & 0.004 \\
\hline $\boldsymbol{E}_{\text {relSD }}$ & 0.037 & 0.008 & 6.337 & 6.249 \\
\hline $\boldsymbol{E}_{\text {relS }}$ & 0.470 & 0.011 & 0.999 & 0.998 \\
\hline $\boldsymbol{E}_{\text {relK }}$ & 2.239 & 0.001 & 1.004 & 1.102 \\
\hline
\end{tabular}

\begin{tabular}{|c|c|c|c|c|}
\hline \multicolumn{5}{|c|}{ Stop time $\left(t_{\text {stop }}\right)$} \\
\hline & $\mathbf{B M}$ & PL & KG & KE \\
\hline$E_{\text {relM }}$ & 0.001 & 0.005 & 0.000 & 0.000 \\
\hline$E_{\text {relSD }}$ & 0.004 & 0.012 & 0.486 & 0.474 \\
\hline$E_{\text {rels }}$ & 0.400 & 0.400 & 0.693 & 0.698 \\
\hline $\boldsymbol{E}_{\text {relK }}$ & 1.498 & 0.237 & 0.808 & 0.941 \\
\hline \multicolumn{5}{|c|}{ Walking speed within aisle ( $\left.S_{\text {aisle }}\right)$} \\
\hline & BM & PL & KG & KE \\
\hline $\boldsymbol{E}_{\text {relM }}$ & 0.000 & 0.001 & 0.000 & 0.001 \\
\hline$E_{\text {relSD }}$ & 0.003 & 0.001 & 0.005 & 0.006 \\
\hline $\boldsymbol{E}_{\text {rels }}$ & 1.021 & 0.019 & 0.024 & 0.015 \\
\hline$E_{\text {relK }}$ & 0.959 & 0.025 & 0.030 & 0.038 \\
\hline
\end{tabular}




\begin{tabular}{|c|c|c|c|c|}
\hline \multicolumn{5}{|c|}{ Response time ( $\left.t_{\text {res2 }}\right)$} \\
\hline & BM & PL & KG & KE \\
\hline$E_{\text {relM }}$ & 0.000 & 0.000 & 0.000 & 0.000 \\
\hline$E_{\text {relSD }}$ & 0.003 & 0.002 & 0.223 & 0.219 \\
\hline$E_{\text {rels }}$ & 1.013 & 0.005 & 0.466 & 0.472 \\
\hline $\boldsymbol{E}_{\text {relK }}$ & 0.919 & 0.156 & 0.573 & 0.091 \\
\hline
\end{tabular}

\begin{tabular}{|c|c|c|c|c|}
\hline \multicolumn{5}{|c|}{ Walking speed downstairs ( $\left.S_{\text {stairs }}\right)$} \\
\hline & BM & PL & KG & KE \\
\hline$E_{\text {relM }}$ & 0.000 & 0.000 & 0.000 & 0.000 \\
\hline$E_{\text {relSD }}$ & 0.004 & 0.003 & 0.000 & 0.002 \\
\hline $\boldsymbol{E}_{\text {rels }}$ & 0.981 & 0.018 & 0.004 & 0.008 \\
\hline$E_{\text {relK }}$ & 0.944 & 0.033 & 0.025 & 0.005 \\
\hline \multicolumn{5}{|c|}{ Response time ( $\left.t_{\text {res4 }}\right)$} \\
\hline & $\mathbf{B M}$ & PL & KG & KE \\
\hline$E_{\text {relM }}$ & 0.000 & 0.001 & 0.002 & 0.001 \\
\hline$E_{\text {relSD }}$ & 0.003 & 0.004 & 5.280 & 5.211 \\
\hline$E_{\text {rels }}$ & 0.999 & 0.028 & 1.063 & 0.988 \\
\hline$E_{\text {relK }}$ & 0.259 & 0.010 & 0.979 & 0.491 \\
\hline
\end{tabular}

Figure 11 compares the weighted relative errors $\left(E_{w_{-}}\right.$rel $)$produced by each PNSM when sampling from the observed variables. The Kernel-Gaussian and the Kernel-Epanechnikov methods are less accurate for tres1, tres3 and tres4 variables with $E_{w \_r e l}>1$ while the Box Muller transform method produces $E_{w_{-} r e l}$ ranged from 0.2 to 0.7 . The Piecewise linear method is most accurate and produces $E_{w_{-} r e l}=0$ for $t_{\text {res1 }}, t_{\text {stop }}$, texit, $S_{\text {aisle }}$ and $S_{\text {stair }}$ variables, $E_{w_{-} \text {rel }}=0.1$ for $t_{\text {res3 }}$ and $t_{\text {res } 4}$ variables and $E_{w_{-} r e l}$ $=0.4$ for $t_{\text {res } 2}$ variable.

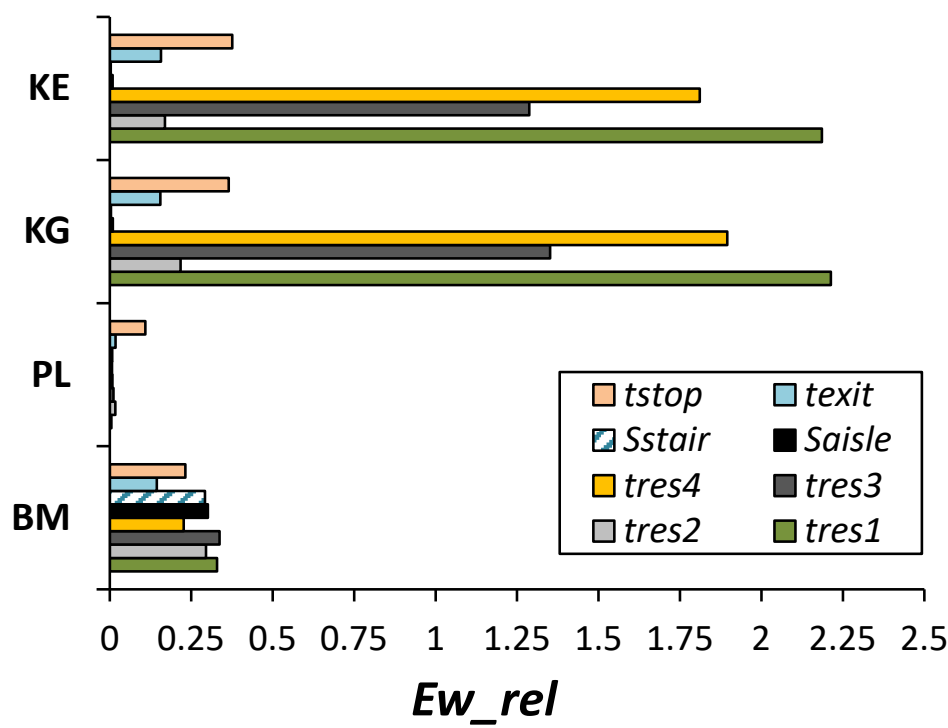

Fig.11. Weighted relative errors produced by the PNSMs when generating samples from the selected variables.

Figure 12 shows the accuracy of the PNSMs according to the type of distribution. As expected, the Kernel-Gaussian and the Kernel-Epanechnikov methods are the most accurate in sampling from normal distributions $\left(E_{w_{-} r e l}=0.06\right.$ and $E_{w_{-} r e l}=0.07$ respectively). Nevertheless, the $E_{w_{-} r e l}$ values produced by these two methods increase dramatically when sampling from uniform and lognormal distributions. The Piecewise linear method is the most accurate in sampling from 
lognormal $\left(E_{w_{-} r e l}=0.04\right)$ and uniform distributions $\left(E_{w_{-} r e l}=0.009\right)$ and it is the only method that produces $E_{w_{-} r e l}$ values below 0.15 for the three types of distributions. On the other hand, the Box Muller method produces $E_{w_{-} r e l}$ values between 0.23 and 0.29 when sampling from the three types of distributions. It is important to note that this method involves two potential sources of error related with 1) the goodness-of-fit and 2) the pseudorandom numbers generation.

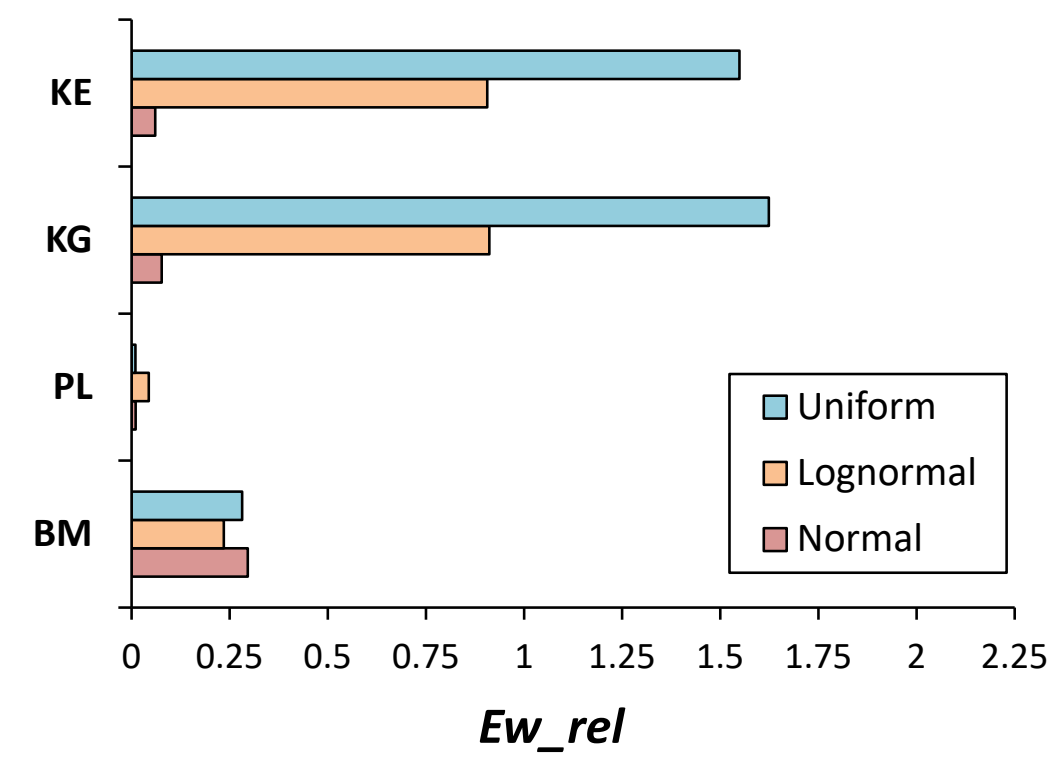

Fig.12. Weighted relative errors produced by the PNSMs when generating samples from normal,

\section{Discussion}

lognormal and uniform distributions.

The evacuation analysis using computer modelling and simulation involves random variables. This work is a novel contribution for assessing degree of exactness of different PNSMs when sampling from evacuation datasets. The method is based on the comparison between experiment and generated distributions. We focus on the statistical parameters as central moments to describe (shape) a given distribution (mean, standard deviation, skewness and kurtosis). It should be noted that this method could be employed to other fire safety engineering applications. The method was applied to eight empirical data samples derived from evacuation experiments and fire drills (3 samples were normally distributed, 3 lognormally distributed and 2 were uniformly distributed).

As expected, the accuracy of the PNSMs varied. The Kernel-Gaussian and the KernelEpanechnikov methods are accurate when sampling from normal distributions but potentially inaccurate when sampling from other distributions. The principal finding of this study is that the Piecewise linear method is the most accurate method for data samples used and showed a better fit with normal, uniform and lognormal distributions. This method is simple and no previous distribution fitting is needed. Therefore, the Piecewise linear is suggested here for evacuation modelling and future developments as long as the model user/developer can have access to empirical (raw) data. Otherwise, analytical methods such as the Box Muller transform method can provide plausible estimations.

Note that this method is likely to produce errors when used for normal and lognormal distributions: 1 ) the error derived from the previous goodness-of-fit test conducted and 2) the error derived from sampling from the distribution. 
Although we feel that the results of the present study are promising, there are some limitations in relation to the number of PNSMs used and the number of datasets studied and their relatively limited sample size. Care should be taken, however, in generalizing from these findings since the use of other (hopefully larger) datasets may lead to different results.

Future research should conduct more tests using the proposed method involving more data samples and PNSMs exploring the generated sample sizes to improve current and future computer evacuation models. For instance, some methods may show a better fit with data-sets of different sizes.

\section{Acknowledgments}

The authors would like to thank the Spanish Ministry of Economy and Competitiveness for the DEFENDER Project grant, Ref.: BIA2015-64866-R, co-financed by ERDS funds.

\section{References}

[1] Averill JD (2010) Five Grand Changes in Pedestrian and Evacuation Dynamics. Pedestrian and Evacuation Dynamics, Springer, New York, pp 1-11

[2] Ronchi E, Reneke PA, Peacock RD (2014) A Method for the Analysis of Behavioural Uncertainty in Evacuation Modelling. Fire Technology 50(6): 1-27

[3] Cuesta A, Abreu O, Alvear D (2016) Future challenges in evacuation modelling, Chapter 5, Evacuation Modeling trends, Springer, New York

[4] Alvear D, Abreu O, Cuesta A, Alonso V (2014) A new method for assessing the application of deterministic or stochastic modelling approach in evacuation scenarios. Fire Safety Journal 65:11-18

[5] PedGo and AENEAS User Manual (2013) TraffGo HT GmbH. http://www.traffgoht.com/downloads/pedestrians/downloads/documents/manual.pdf

[6] STEPS Simulation of Transient and Pedestrian movementS: User Manual, unpublished, available with egress model from Mott MacDonald. http://www.mottmac.com.

[7] Pathfinder Technical Reference (2016) Thunderhead Engineering. http://www.thunderheadeng.com/downloads/pathfinder/tech_ref.pdf

[8] Exit 89 (2013) ISO/TR 10796-4 Fire Safety Engineering - Examples on Verification and Validation of a Calculation Method - Part 5: Egress Model. https://www.iso.org/obp/ui/\#iso:std:iso:tr:16730:-5:ed-1:v1:en

[9] Bensilium M, Purser D (2003) GridFlow: an Object-Oriented Building Evacuation Model Combining Pre-movement and Movement Behaviours for Performance-Based Design. Fire Safety Science 7: 941-952. http://www.iafss.org/publications/fss/7/941/view

[10] Korhonen T, Hostikka S (2009) Fire Dynamics Simulator with Evacuation: FDS+Evac: Technical Reference and User's Guide. VTT Technical Research Centre of Finland, VTT Working Papers 119. http://lovreglio.altervista.org/documentation.htm

[11] Egress: Simulex User Guide (2014) IES Virtual Environment. file://C:/Users/gidai\%2012/Downloads/simulex\%20(1).pdf

[12] BuildingEXODUS User Guide (2011) unpublished, available with egress model from Fire Safety Engineering Group, University of Greenwich. http://fseg.gre.ac.uk/exodus/

[13] MassMotion Flow Manual (2015) Oasys Software Limited. http://www.oasyssoftware.com/media/Manuals/Latest_Manuals/MassMotion_Flow.pdf

[14] VisWalk User Manual (2015) PTV Group. http://cgi.ptvgroup.com/cgibin/en/traffic/vissim_download.pl 
[15] Gwynne SV, Boyce K (2016) Engineering Data. In: SFPE Handbook of Fire Protection Engineering, $5^{\text {th }}$ edn. Cambridge, UK, pp 2429-2551

[16] Cuesta A, Gwynne S (2016) The collection and compilation of school data for model use. Safety Science 84:24-36

[17] Cuesta, A. et al. (2016) School egress data: comparing the configuration and validation of five egress modelling tools, Fire and Materials, DOI: 10.1002/fam.2405

[18] Gwynne S, Galea ER, Parke J and Hickson J (2003) The collection and analysis of preevacuation times derived from evacuation trials and their application to evacuation modelling. Fire Technology 39:173-195

[19] BSI, PD 7974-6:2004 (2004) The application of fire safety engineering principles to fire safety design of buildings. Part 6: Human factors: Life safety strategies - Occupant evacuation, behaviour and conditions (Sub-system 6). British Standards Institute

[20] ISO/TR 16738: 2009 (2009) Fire-safety Engineering- Technical Information on Methods for Evaluating Behaviour and Movement of People, Switzeland

[21] MSC.1/Circ.1238 (2007) Guidelines for Evacuation Analysis for New and Existing Passenger Ships, IMO International Maritime Organization

[22] Lord, J. et al. (2005), Guide for evaluating the predictive capabilities of computer egress models, NIST GCR 06-886

[23] Lovreglio, R., Ronchi, E. and Borr, D. (2014). The Validation of Evacuation Simulation Models through the Analysis of Behavioural Uncertainty. Reliability Engineering \& System Safety 131:166-174

[24] Knuth, D. E. (1998). The art of computer programming, 3rd ed. seminumerical algorithms, vol. 2.

[25] Cornejo Díaz, N., Vergara Gil, A. and Jurado Vargas, M. (2010). Assessment of the suitability of different random number generators for Monte Carlo simulations in gammaray spectrometry. Applied radiation and isotopes 68:469-473.

[26] Rukhin, A. et al. (2010). A Statistical Test Suite for Random and Pseudorandom Number Generators for Cryptographic Applications. NIST, Special Publication 800-22.

[27] Doganaksoy, A., Ege, B., Koçak, O., \& Sulak, F. (2010). Cryptographic Randomness Testing of Block Ciphers and Hash Functions. IACR Cryptology ePrint Archive, 2010, 564.

[28] Capote JA, Alvear D, Abreu O, Cuesta A (2012) Analysis of evacuation procedures in high speed train fires. Fire Safety Journal 49:35-46

[29] Capote JA, Alvear D, Abreu O, Cuesta A, Alonso V (2012) A stochastic approach for simulating human behaviour during evacuation process in passenger trains. Fire Technology, 48 (4): 911-925

[30] Cuesta A, Abreu O, Alvear D (2016) Methods for measuring collective behaviour in evacuees. Safety Science 88:54-63

[31] NIST/SEMATECH e-Handbook of Statistical Methods. http://www.itl.nist.gov/div898/ handbook 13

[32] D’Agostino RB, Belanger A (1990) A suggestion for using powerful and informative tests of normality. Am Statist 44(4):316-321 14

[33] Rahman M, Pearson LM, Heien HC (2006) A modified Anderson-Darling test for uniformity. Bull Malays Math Sci Soc 29(1):11-16

[34] Box GEP, Muller ME (1958) A Note on the Generation of Random Normal Deviates, The Annals of Mathematical Statistics 29(2): 610-611

[35] Thomas, D. B, Luk, W and Villasenor, J.D (2007), Gaussian Random Number Generators, ACM Computing Surveys, Vol. 39(4): 11-11:38 
[36] Marsaglia, G and Bray, T.A. (1964) A Convenient Method for Generating Normal Variables, SIAM Review, Vol. 6 (3): 260-264

[37] Marsaglia, G and Tsang, W.W. (2000) The Ziggurat method for generating random variables, Journal of Statistical Software, Vol. 5(8): 1-7

[38] Hamann B, Chen JL (1994) Data point selection for Piecewise Linear Curve approximation, Computer Aided Geometric Design 11 (3): 289

[39] Epanechnikov VA (1969) Non-parametric Estimation of a Multivariate Probability Density, Theory Probab. Appl. 14(1): 153-158

[40] Silverman BW (1998) Density Estimation for Statistics and Data Analysis. Chapman \& Hall/CRC, London 